\title{
Extracellular Pax6 Regulates Tangential Cajal-Retzius Cell Migration in the Developing Mouse Neocortex
}

\author{
H. Kaddour ${ }^{1,2,5,6}$, E. Coppola ${ }^{2,5,6}$, A. A. Di Nardo ${ }^{1, \dagger}$, C. Le Poupon ${ }^{1}$, P. Mailly ${ }^{3}$, \\ A. Wizenmann ${ }^{4}$, M. Volovitch ${ }^{1}$, A. Prochiantz ${ }^{1, \ddagger}$ and A. Pierani ${ }^{2,5,6}$
}

${ }^{1}$ Center for Interdisciplinary Research in Biology, Centre National de la Recherche Scientifique Unité mixte de recherche 7241/Institut national de la santé et de la recherche médicale U1050, Paris Science Lettre University, Labex MemoLife, Collège de France, 11 place Marcelin Berthelot, Paris 75231, France ${ }^{2}$ Institut Jacques Monod, Centre National de la Recherche Scientifique Unité mixte de recherche 7592, Université Paris Diderot, Sorbonne Paris Cité, 15 Rue Hélène Brion, Paris 75013, France ${ }^{3}$ Center for Interdisciplinary Research in Biology, Centre National de la Recherche Scientifique, Core Facility Orion, 11 Place Marcelin Berthelot, Paris 75231, France ${ }^{4}$ Department of Anatomy, Institute of Clinical Anatomy and Cell, University of Tübingen, Osterbergstrasse 3, Tübingen 72074, Germany ${ }^{5}$ Current address: Imagine Institute for Genetic Diseases, Université Paris Descartes, 24 Boulevard du Montparnasse, Paris 75015, France ${ }^{6}$ Current address: Institute of Psychiatry and Neuroscience of Paris, Université Paris Descartes, 102-108 Rue de la Santé, Paris 75014, France

Address correspondence to Alessandra Pierani, Imagine Institute for Genetic Diseases, Université Paris Descartes, 24 Boulevard du Montparnasse, Paris 75015, France/Institute of Psychiatry and Neuroscience of Paris, Université Paris Descartes, 102-108 Rue de la Santé, Paris 75014, France and Alain Prochiantz, Center for Interdisciplinary Research in Biology, Centre National de la Recherche Scientifique Unité mixte de recherche 7241/Institut national de la santé et de la recherche médicale U1050, PSL University, Labex MemoLife, Collège de France, 11 Place Marcelin Berthelot, Paris 75231, France. Email: alessandra.pierani@inserm.fr; alain.prochiantz@college-de-france.fr

${ }^{\dagger}$ A. A. Di Nardo, http://orcid.org/0000-0003-2944-488X

‡A. Prochiantz, http://orcid.org/0000-0001-7626-5863

\begin{abstract}
The embryonic mouse cortex displays a striking low caudo-medial and high rostro-lateral graded expression of the homeoprotein transcription factor Pax6, which presents both cell autonomous and direct noncell autonomous activities. Through the genetic induction of anti-Pax6 single-chain antibody secretion, we have analyzed Pax6 noncell autonomous activity on the migration of cortical hem- and septum-derived Cajal-Retzius (CR) neurons by live imaging of flat mount developing cerebral cortices. Blocking extracellular Pax6 disrupts tangential CR cell migration patterns by decreasing the distance traveled and changing both directionality and depth at which CR cells migrate. Tracking of single CR cells in mutant cortices revealed that extracellular Pax6 neutralization enhances contact repulsion in medial regions yet reduces it in lateral regions. This study demonstrates that secreted Pax 6 controls neuronal migration and distribution and suggests that it acts as a bona fide morphogen at an early stage of cerebral cortex development.
\end{abstract}

Key words: Cajal-Retzius neurons, embryonic cerebral cortex, migration, noncell autonomous, Pax6

๑) The Author(s) 2019. Published by Oxford University Press. All rights reserved. For permissions, please e-mail: journals.permission@oup.com. 


\section{Introduction}

Cajal-Retzius (CR) cells are a transient glutamatergic neuron population residing at the surface of the developing cerebral cortex (Marin-Padilla 1998). They comprise molecularly distinct subtypes born in three different progenitor domains of the mouse cortical neuroepithelium: the cortical hem $(\mathrm{CH})$, the septum (S), and the ventral pallium at pallial-subpallial boundary (Bielle et al. 2005; Yoshida et al. 2006; Tissir et al. 2009). Between embryonic days 10.5 (E10.5) and 12.5, CR subtypes migrate tangentially within the preplate to specifically populate the caudo-medial (CM), rostro-medial (RM), and lateral cortex (Bielle et al. 2005; Garcia-Moreno et al. 2007; Griveau et al. 2010; Villar-Cervino et al. 2013). CR cell migration and distribution at the surface of the neocortex play important roles in controlling the size and positioning of primary and higher-order cortical areas (Griveau et al. 2010; Barber et al. 2015). It has been proposed that CR cells act as mobile signaling cells that participate in the production and maintenance of morphogen gradients required to coordinate neurogenesis and to delineate areal boundaries within the cortical ventricular zone (VZ; Griveau et al. 2010).

At the molecular level, transcription factors play key functions in the early regionalization of the cortex (Brunet et al. 2007; O'Leary et al. 2007). Among them, the homeoprotein (HP) family has been the focus of several theoretical and experimental studies showing that they have essential functions in the positioning of boundaries within the developing cortex and, beyond, in the entire nervous system. In particular, Pax6 and Emx2 position the boundary between the primary visual and somatosensory territories (O'Leary et al. 2007). A link between Pax6 and the control of CR cell migration was suggested by previous studies where accumulation of CR cells in the rostral pallium (Matsuo et al. 1993; Stoykova et al. 2003) and abnormal migration of CH-CR cells was detected in Sey mutant embryos (Ceci et al. 2010). Up to now, the role of His in early cortical regionalization was thought to be through intrinsic mechanisms in progenitor cells. However, many HPs, including Pax6, can be secreted and internalized (Prochiantz 2000; Joliot and Prochiantz 2004) and thus display a noncell autonomous function, which has led to the hypothesis that part of their morphogenetic activity might involve their secretion (Holcman et al. 2007; Prochiantz and Di Nardo 2015; Quininao et al. 2015; Di Nardo et al. 2018).

In support of the latter hypothesis, Pax6 was found at the cell surface of ventral neural tube tissues where it regulates the migration of oligodendrocyte precursors (Di Lullo et al. 2011), and the graded expression of extracellular Engrailed, another HP, is a guidance cue for retinal growth cones navigating at the surface of the optic tectum (Brunet et al. 2005; Wizenmann et al. 2009). We therefore investigated whether Pax 6 regulates $C R$ cell migration via a noncell autonomous activity. To specifically trigger removal of extracellular Pax6 we used a mouse model harboring the sequence for an inducible single-chain antibody (scFv) directed against Pax6 in the ROSA26 locus (Bernard et al. 2016). We demonstrate that neutralizing Pax6 in the extracellular space modifies the migration of $\mathrm{CH}-$ and $\mathrm{S}-\mathrm{CR}$ cells.

\section{Materials and Methods}

\section{Animals}

scFuPax6 (Bernard et al. 2016) and $\Delta$ Np73CrelRESGFP (Tissir et al. 2009) transgenic mice were kept in a C57BL/6J background. The day of vaginal plug was considered to be E0.5. All animal procedures, including housing, were carried out in accordance with the recommendations of the European Economic Community (86/609/EEC), the French National Committee (87/848), and French bylaws (AGRG1240332A/AGRG1238724A/AGRG1238767A/ AGRG1238729A/AGRGR1238753A). All mouse work was approved by the Veterinary Services of Paris (authorization number: 751454) and by the Animal Experimentation Ethical Committee Buffon (CEEA-40) (reference: CEB-34-2012).

\section{Whole Flattened Cortices Culture}

Whole flattened cerebral cortices cultures were adapted from Barber et al. (2015). Cortices were dissected from E11.5 $\Delta \mathrm{Np} 73^{\mathrm{Cre} /+}$

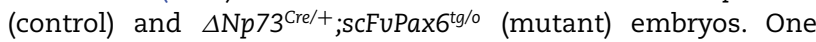
single incision was made to remove the ventral subpallial tissue. Explants were cultured for $5 \mathrm{~h}$ before time-lapse acquisition on a Millicell permeable membrane (pore size, $0.4 \mu \mathrm{m}$; Millipore, Molsheim, France) with the ventricular side facing the membrane, in phenol red-free high-glucose Dulbecco's Modified Eagle Medium (DMEM) (Sigma, Lyon, France) containing B27 supplement (Gibco, Paisly, Scotland) and penicillin/streptomycin antibiotics $\left(100 \mu \mathrm{g} / \mathrm{mL}\right.$; Sigma) in $5 \% \mathrm{CO}_{2}$ at $37^{\circ} \mathrm{C}$. Shortly before imaging, the flattened cortex was transferred with the Millicell membrane and inverted onto a glass-bottom microscope imaging chamber with the pial surface directly adjacent to the glass. Explants were mounted in purified bovine collagen gel $(25 \mu \mathrm{L}$ /explant; Advanced Biomatrix, San Diego, CA), which polymerizes in $15 \mathrm{~min}$ at $37^{\circ} \mathrm{C}$. Culture conditions did not change the percentage of tissue occupied by Reelin (Reln; pial surface) and Pax6 (VZ) staining in DL regions between 0 and $8 \mathrm{~h}$, both in control and mutant cortices (Supplementary Figure S4 and data not shown).

\section{D Time-Lapse Acquisition}

Time-lapse acquisition of migrating CR cells was performed $>8 \mathrm{~h}$ in an incubation chamber at $37^{\circ} \mathrm{C}$ and $5 \% \mathrm{CO}_{2}$ in phenol redfree DMEM solution by using an inverted laser scanning confocal microscope (Zeiss 780) with an oil immersion $25 \times$ objective. EGFP protein was excited at $488 \mathrm{~nm}$ and images were acquired at 500-550 nm. Depending on explant size, 4D time-lapse images were generated by acquiring automatically 9-14 optical $Z$ sections with a $z$-step size of $8 \mu \mathrm{m}$ every $30 \mathrm{~min}$, and $70-140$ focal tiles were merged to visualize the entire flattened cortex with a $512 \times 512$ pixel resolution.

\section{Bioinformatics Processing and Movie Analysis}

Successive steps were used to disassemble and divide resulting movies, exceeding $15 \mathrm{~GB}$, in time and Z points successively by using the Zeiss Zen software (Carl Zeiss MicroImaging, Marlyle-Roi, France) and the ImageJ software to convert images in 3D tiff stacks. 4D reconstruction and display of $C R$ cell migration were built up by Imaris software (Bitplane; version 8.2.1, Zurich, Switzerland). $\mathrm{GFP}^{+}$cell tracks were obtained from four flattened brains per genotype (control or mutant). Using the spot cell-tracking module, cells were spatially classified depending on their genotype and origins ( $\mathrm{CH}$ or $\mathrm{S}$ ) and tracked manually over time and along the medio-lateral, rostro-caudal, and depth axes. We selected CR cells migrating in the caudal quadrant of the explant as corresponding to $\mathrm{CH}-\mathrm{CRs}$ cells and those migrating in the rostral quadrant as S-CR cells from four embryos per genotype harvested from different mothers (according to Barber et al. 2015). We excluded from the analysis 
CR cells migrating at middle levels along the rostro-caudal axis for which the origin could not be ascertained. A total of 1478 tracks were analyzed, 726 control cells and 752 mutant cells. The directionality index was calculated by dividing the Euclidean distance (the shortest distance between the start and end position of a cell trajectory) by the actual path length followed by the cells during migration. The overall directionality in migration was determined for each region of interest by automatic clustering tracks to a common origin using the Imaris transpose tracks function. In Figure $4 \mathrm{E}$, a mean displacement vector along the medio-lateral and rostro-caudal axes was calculated for each region and represented in a $2 \mathrm{D}$ vectorial projection clustered at a common origin. CR cell behaviors upon contact were subdivided and analyzed based on previous strategies (Villar-Cervino et al. 2013; Barber et al. 2015). Briefly, contacts between two cells were scored positive if occurring within a single optical slice from $\triangle \mathrm{Np} 73^{\mathrm{Cre} /+}$ (control) and $\triangle \mathrm{Np} 73^{\mathrm{Cre} /+} ; \mathrm{scFuPax} 6^{\mathrm{tg} / \mathrm{o}}$ (mutant) explants in the rostral and caudal regions. The angle of deviation was observed using the Imaris cell-tracking spot module and considered contact with repulsion if cells changed trajectory with an angle of $>90^{\circ}$.

\section{Cell Surface Biotinylation}

Biotinylation on whole cortex was performed according to manufacturer instructions (PIERCE kit: 89881). Cortices of E11.5 mice were dissected and treated with biotin in the presence or absence of NP40 (0.02\%) for $10 \mathrm{~min}$ on a rocking platform. Samples were washed several times in Phosphate buffered saline (PBS)-glycine and disrupted by sonication on ice. Proteins were extracted, loaded on streptavidin beads, eluted, and analyzed by western blot with Anti-Pax6 (rabbit monoclonal; 1/500; Covance, London, UK) and anti-RhoA (mouse monoclonal; 1/200; Santa Cruz, Biotechnology, Heidelberg, Germany). Membranes were imaged with a LAS-4000 gel imager (Fujifilm), and band intensity was quantified by densitometry with ImageJ on 9-10 independent embryos for each condition.

\section{Tissue Preparation and Immunohistochemistry}

Embryos and flattened cortices were fixed by immersion in $4 \%$ paraformaldehyde (PFA), PBS for $2 \mathrm{~h}$ at $4^{\circ} \mathrm{C}$, and rinsed in PBS for $1 \mathrm{~h}$. Samples were cryoprotected overnight in $20 \%$ sucrose, PBS, and embedded in O.C.T. compound (Sakura). Embedded tissues were sectioned on a cryostat with $14 \mu \mathrm{m}$ steps for embryonic brains and $20 \mu \mathrm{m}$ for flattened cortices. Immunohistochemistry on sections was performed as previously described (Pierani et al. 2001). Primary antibodies were rabbit anti-Pax6 (Biolegends; 1:500, San Diego, CA), G10 mouse anti-Reln (Calbiochem; 1:1000, San Diego, CA), and rabbit anti-GFP (Molecular Probes; 1:1000, Waltham, MA). Nuclei were counterstained using FluoromountG with 4',6-Diamidino-2-phenylindole (DAPI) (Thermo Fisher Scientific, Waltham, MA).

\section{Imaging and Cell Counting}

Images were acquired with a Zeiss Spinning Disk W1 microscope. Coronal cryostat sections of E11.5 embryos brains and flattened cortices were quantified with images from rostral and caudal sections with at least three brains analyzed for each genotype. The cortex was subdivided into medial (M) and dorsolateral (DL) domains and cells were quantified using the cellcount tool with Image J software.

\section{Statistics}

Prism 6 software (GraphPad; version 6.01) was used for statistical analysis. When data followed a normal distribution, paired comparisons were analyzed with t-test, whereas multiple comparisons were analyzed using one-way analysis of variance (ANOVA) with post hoc Bonferroni correction. Data are presented as mean and \pm SEM throughout the manuscript. $P<0.05$ was considered significant.

\section{Results}

\section{Following CR Trajectories in 4D}

Extracellular localization of Pax6 has already been demonstrated in the chick spinal cord (Di Lullo et al. 2011). To confirm that this is also the case in the mouse developing cerebral cortex, E11.5 cortices were immunostained for Pax6 and images clearly showed extranuclear localization of Pax6 (Supplementary Fig. S1A,B). Furthermore, embryos were dissected and extracellular proteins were biotinylated and purified on streptavidin beads. Pax6 was indeed found in the extracellular compartment, while intracellular protein RhoA was not accessible to biotinylation in the absence of NP-40 (Supplementary Fig. S1C,D). To specifically follow $\mathrm{CR}$ cells generated at the level of the $\mathrm{CH}$ and S (CH-CR cells and S-CR cells, respectively), we imaged E11.5 flattened cortices in culture from the $\Delta \mathrm{Np} 73^{\mathrm{Cre}}$ mouse line in which Cre and EGFP are specifically expressed in these two cell populations (Tissir et al. 2009; Fig. 1A). To compare CR migration in a wild-type environment or upon Pax6 extracellular neutralization (thereafter, mutant), single-chain secreted antiPax6 antibody (Pax6 scFv) was induced in CH-CR cells and S-CR cells by crossing $\triangle \mathrm{Np} 73^{\mathrm{Cre}}$ and scFuPax 6 mouse lines (Tissir et al. 2009; Bernard et al. 2016). The Pax6 scFv was characterized in earlier studies (Lesaffre et al. 2007; Di Lullo et al. 2011; Bernard et al. 2016). It is specific for Pax6 and devoid of a significant cell-autonomous activity, even when expressed without signal peptide, likely because of the instability of disulfide bonds in the reducing cytoplasmic milieu (Lesaffre et al. 2007). Moreover, Pax6 is specifically expressed by radial glia (RG) progenitors that do not undergo recombination using these Cre line allowing to exclusively test the role of extracellular Pax6 on CR cell behavior.

Four cortices were used for each genotype (wild-type and mutant) to track $>700 \mathrm{CR}$ cells during $8 \mathrm{~h}$ in order to obtain $4 \mathrm{D}$ views of cell migration (Fig. $1 A, B$; movie S6). Initial cell positions were not exactly the same in each cortex regardless of genotype, reflecting slightly different embryonic stages (Fig. 1B). Indeed, development proceeds very rapidly and CR cells generated between E10.5 to E11.0 cover the entire pallium within $12 \mathrm{~h}$ (Bielle et al. 2005; Griveau et al. 2010). We reasoned that if the effect of blocking extracellular Pax6 is robust enough, it should be visible regardless of this inter-cortical heterogeneity in initial cell position, and thus we first performed a global analysis before subdividing cortices into several compartments.

\section{Extracellular Pax6 Influences CR Cell Migration Trajectories}

To compare CR subtypes in the different cortices, we imposed a theoretical migration starting point for all cells in order to analyze each cortex separately (Fig. 2A) and to pool the data for each condition (Fig. 2B). We first qualitatively confirmed that cell 
A
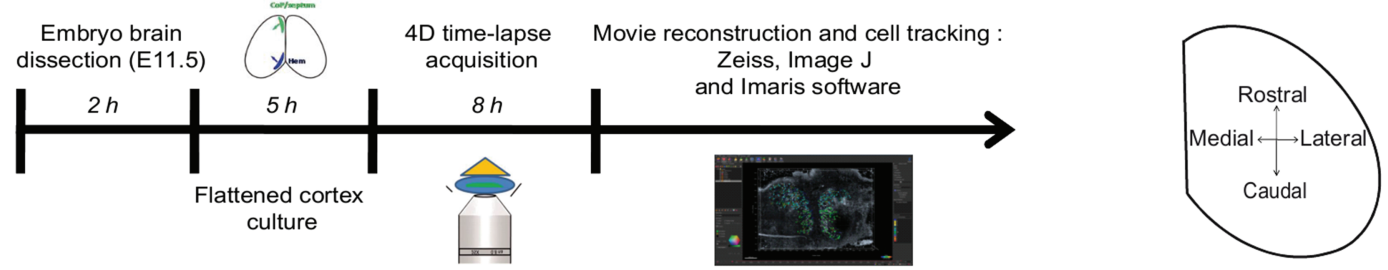

B
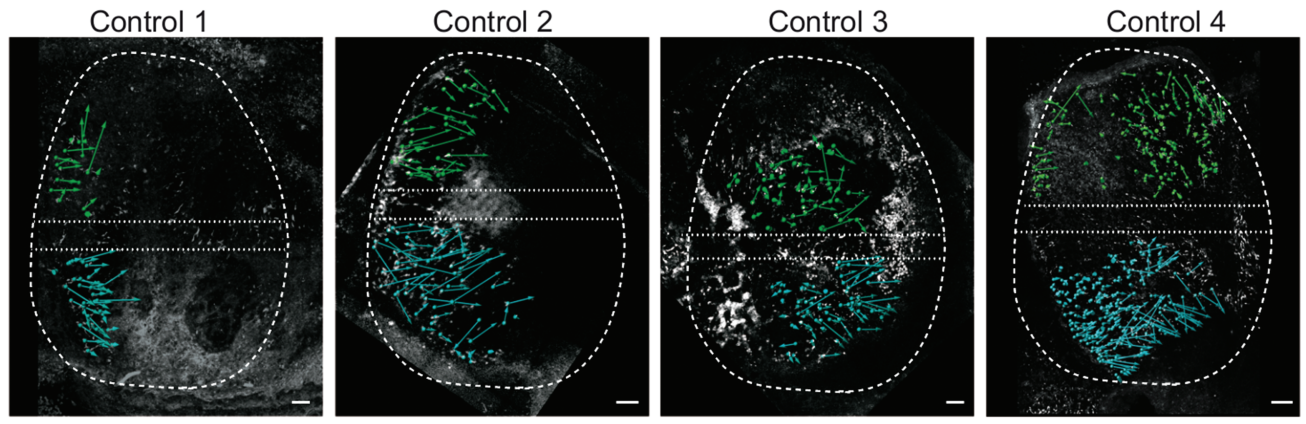

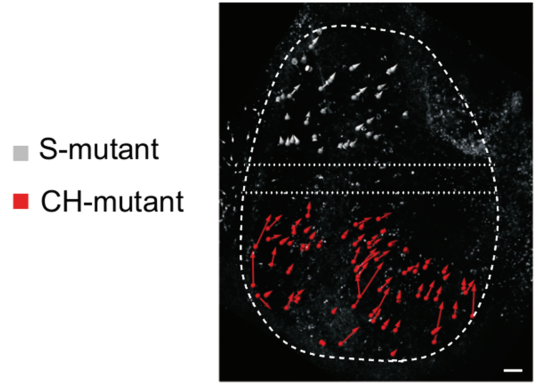

Mutant 1

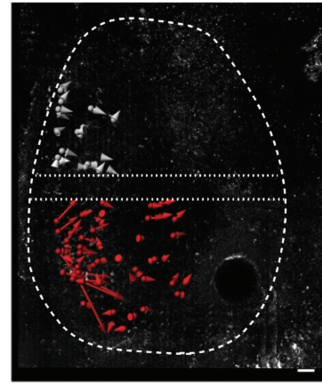

Mutant 2

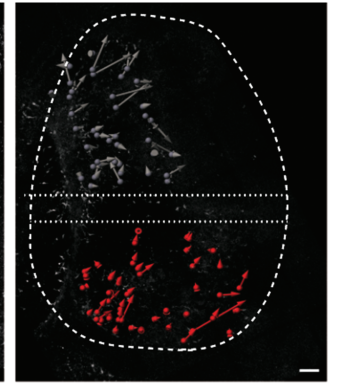

Mutant 3

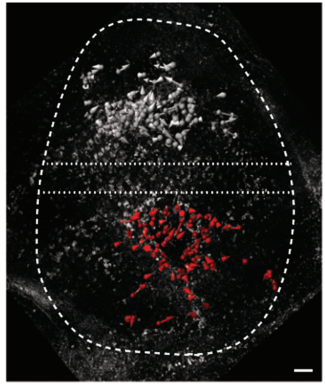

Mutant 4

Figure 1. 4D acquisition of CR cell migration patterns. (A) Overview of the successive steps to record and analyze the migration of GFP + CR cells. (B) Cell tracks from $8 \mathrm{hr}$ live imaging of S- and CH-CR cells in E11.5 flattened cortices of $\triangle \mathrm{Np} 73^{\mathrm{CreIRESGFP}}$ (control) and $\triangle \mathrm{Np} 73^{\mathrm{CreIRESGFP}}$;scFuPax $6^{\mathrm{tg} / \mathrm{o}}$ (mutant) embryos. Tracks are represented by displacement vectors. The CR cells ( $\mathrm{S}$ or $\mathrm{CH}$ ) for which origin could not be ascertained were excluded from the images, and their localization was delimited by dashed lines. Scale bar: $100 \mu \mathrm{m}$.

fitness was preserved during the $8 \mathrm{~h}$ of image acquisition by following the progression of migration during four successive time intervals in pooled cortices (Supplementary Fig. S2A). In control and mutant conditions for both CR subtypes, track path length increased progressively suggesting that cell migration globally continued up to the end of the imaging period. Furthermore, for both CR subtypes, the mean speed after $480 \mathrm{~min}$ of recording showed no differences between control and mutant cells suggesting that blocking extracellular Pax6 did not affect their ability to migrate (Supplementary Fig. S2B).

We next analyzed the migration pattern of CR cells after $480 \mathrm{~min}$ of recording, in each separate cortex (Fig. 2A) and pooled cortices (Fig. 2B), by quantifying the trajectories along the medio-lateral and the rostro-caudal axes (Fig. $2 B$ and Supplementary Fig. S3). Compared to controls, both mutant S- and $\mathrm{CH}-\mathrm{CR}$ cells presented shorter track path length with a lower directionality index indicating that mutant $C R$ neurons migrate along less linear trajectories (Fig. 2A-C). The majority of $\mathrm{S}-$ and $\mathrm{CH}-\mathrm{CR}$ cells in controls migrated toward lateral regions with a preference for the rostro-lateral (RL) direction (35.6\% and 51.3\%, respectively) (Fig. 2B). In mutants, compared to controls, the percentage of $\mathrm{S}-\mathrm{CR}$ cells migrating in RL directions increased $(45.5 \%$ vs $35.6 \%$, respectively; Fig. 2B) whereas that of $\mathrm{CH}-\mathrm{CR}$ cells migrating caudo-laterally (CL) was preferentially enhanced $(35.4 \%$ vs $21.1 \%$, respectively; Fig. 2B). To further examine these differences, we analyzed distances traveled by $C R$ cells in the RL and CL directions (Fig. 2D,E and Supplementary Fig. S3). Extracellular Pax6 neutralization was efficient in decreasing path length of SCR cells migrating rostro-laterally (39.7\%) with a smaller but significant effect on Euclidean distance (21.6\%) defined as the shortest distance between the initial and final positions in cell trajectory. Notably, no differences were observed for S-CR cells migrating CL. For $\mathrm{CH}-\mathrm{CR}$ cells, Pax6 neutralization resulted in a reduction of both path length and Euclidean distance values whether they migrated RL or CL $(25.6 \%$ vs $41.8 \%$ and $15.3 \%$ vs $32.0 \%$, respectively). A representative trajectory of CR cells in each condition (Fig. 2D,E) illustrates that neutralizing extracellular Pax6 modifies the migration of S-CR and $\mathrm{CH}-$ $\mathrm{CR}$ neurons toward the lateral part of the developing cortex with a stronger decrease in overall Euclidean distance than total path length. Together these results suggest that secreted Pax6 influences more strongly directionality than the ability to migrate. 


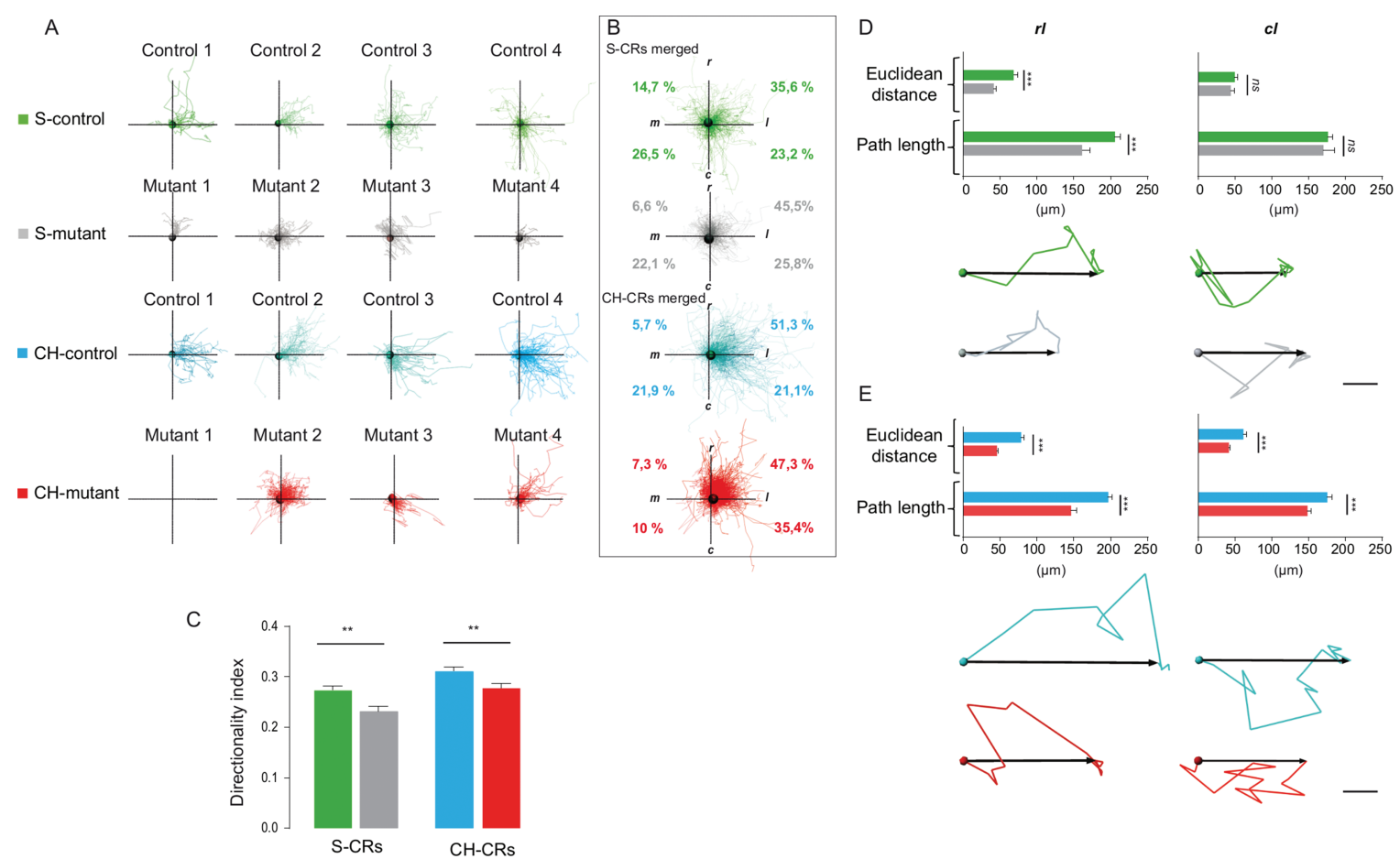

Figure 2. Noncell autonomous activity of Pax6 on CR cell trajectories and directionality. (A and B) Tracks of migrating S- and CH-CR cells (A) in each control and mutant cortex after 480 min along the M-lateral and rostro-caudal axes. The tracks are pooled in (B) and the distribution of tracks per quadrant is shown by percentage. (C) Directionality index is the ratio of Euclidean distance to path length. (D and E) Path length and Euclidean distance traveled by CR cells in the RL and CL quadrants. Representative trajectories of S- and CH-CRs are illustrated for each condition. Scale bar: $10 \mu \mathrm{m}$. Values are mean \pm SEM; ANOVA: ns means not statistically different; ${ }^{* *} \mathrm{P}<0.01 ; * * \mathrm{P}<0.001$.

\section{Position-Dependency of Noncell Autonomous Pax6 Activity}

To study whether the effect of extracellular Pax6 neutralization depends on the region of the developing cortex where CR cells migrate, we took into account the differences in CR cell track start position. Cortices were subdivided into four compartments along the medio-lateral and the rostro-caudal axes with cell numbers ranging from 97 to 265 per compartment to allow for statistical analyses. The two rostral territories (RM and RL) are enriched in S-CR cells, while the caudal ones (CM and CL) primarily contain $\mathrm{CH}-\mathrm{CR}$ cells. In addition, $\mathrm{RM}$ and $\mathrm{CM}$ compartments are closer to the CR cell sources than RL and CL compartments, which abut the "arrival regions". CR migration was analyzed separately for the four compartments (Fig. 3A-D). In all four compartments both S- and CH-CR cells in mutants traveled shorter Euclidean distances along the medio-lateral axis with respect to controls. However, the rostro-caudal axis migration of both CR subtypes was only influenced by Pax6 neutralization in the lateral compartments where both S- and CH-CR cells appeared to travel shorter Euclidean distances and to move less along this axis (Fig. $3 C, D$ ). The mean directionality vectors of controls and mutants (Fig. $3 E$ ) summarize the effect of blocking extracellular Pax6 on trajectories in the different territories. They show that travel distance is reduced for mutant CR cells in all four compartments and that the direction of migration is more highly affected in the lateral cortex, where SCR cells show increased migration in the rostral direction. Such effects predict changes in CR cell positioning in vivo. We thus analyzed CR distribution on sections by counting GFP ${ }^{+}$CR cells (Fig. 4A) in the DL and M domains at E11.5 (Fig. 4B,C). In mutants, an increase in the number of $\mathrm{GFP}^{+}$cells was observed in $\mathrm{DL}$ regions at both rostral and caudal levels, whereas in $\mathrm{M}$ regions cell numbers increased at rostral levels but decreased in the CM region (Fig. $4 D, E$ ). The accumulation of CR cells in the mutant rostral ( $\mathrm{M}$ and DL regions) and DL caudal levels is consistent with time-lapse observations and a redirection of the cells toward more rostral territories.

Taken together, these results show that neutralization of extracellular Pax6 globally reduces the distance traveled by $\mathrm{CR}$ cells and redirects them toward the rostral direction when reaching the end of migration in the lateral cortex.

\section{Blocking Extracellular Pax6 Affects the Depth of CR Cell Migration}

Previous studies have analyzed migration parameters of CR cells in 2D or 3D environments (Ceci et al. 2010; Villar-Cervino et al. 2013; Barber et al. 2015). Migration within the depth of the tissue has never been analyzed in whole flattened cortical preparations because of the excessive size of the movies. We have now devised a method to analyze over 15 GB time-lapse files by movie frame separation and reconstruction (see Materials and Methods). We were thus able to quantify the effect of Pax6 neutralization on S- and CH-CR localization within the depth (Zaxis) of the developing cortex as a function of time in the four compartments of interest (Fig. 5). Although percentages along the $\mathrm{Z}$-axis might not represent real in vivo values due to tissue shrinking in flattened cortex cultures, our data demonstrate that close to CR sources (RM and CM domains) S- and CH-CR cells in controls migrated at $40 \%$ or $30 \%$ depth, respectively, with 

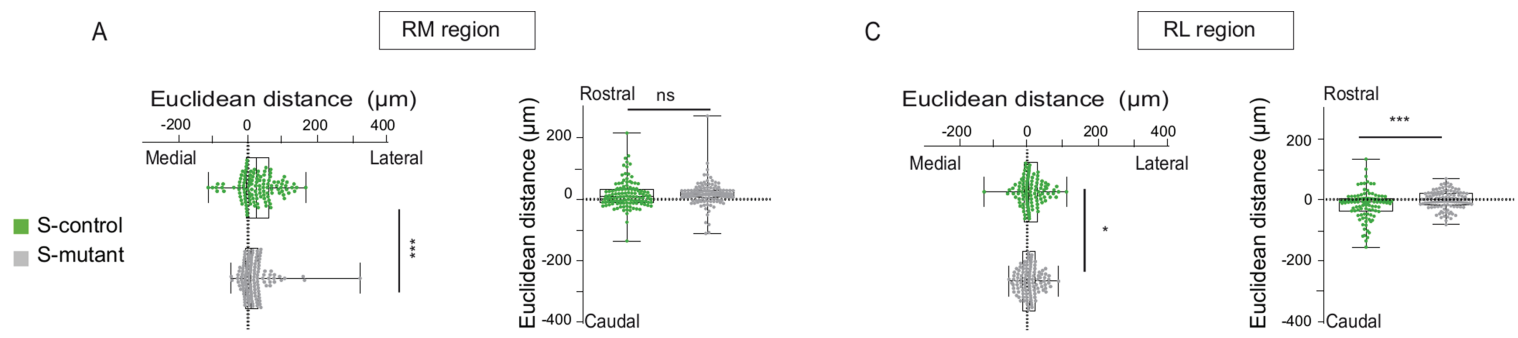
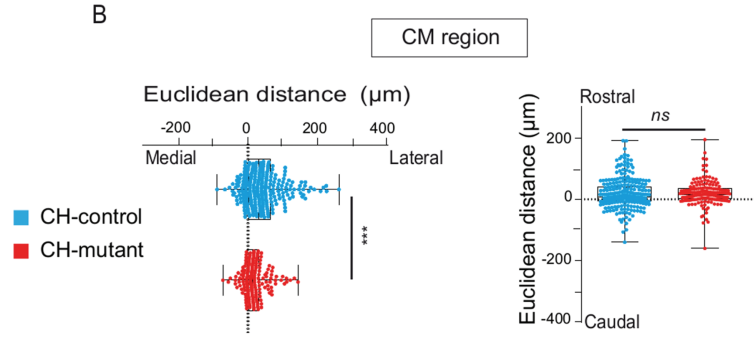

$E$

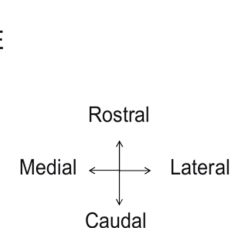

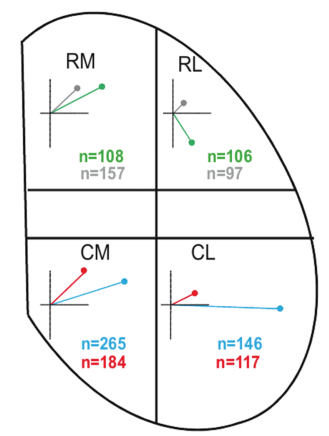

Figure 3. Position dependency of noncell autonomous Pax6 activity. (A-D) S- and CH-CRs migration (Euclidean distance) in the four compartments: RM (A), CM (B), RL $(C)$, and CL (D) for control and mutant cortices. Each point represents a cell. (E) Control and mutant cortices were subdivided in two compartments along the mediolateral axis and in three compartments along the rostro-caudal axis. In the four select compartments, mean displacement vectors were calculated from CR cell tracks. Vectors represent the Euclidean distance traveled during $8 \mathrm{~h}$ of migration. A total of 625 and 555 CRs were tracked in control and mutant cortices, respectively. Values shown are mean \pm SEM; ANOVA: $n s$ means not statistically different; ${ }^{*} P<0.05 ;{ }^{* * *} P<0.001$

a wider dispersion between the ventricular side and the pial surface in the mutant CH-CR cells (Fig. 5A). When closer to the end of their migration (RL and CL territories), $\mathrm{CH}-\mathrm{CR}$ cells in controls spread more along the depth compared to S-CR cells which migrated at $\sim 50 \%$ depth. This behavior was lost in the mutant environment where both $\mathrm{S}-$ and $\mathrm{CH}-\mathrm{CR}$ cells migrated close to the pial surface (Fig. 5B). Representative images of the different regions of interest (Fig. 5A,B) demonstrate that blocking extracellular Pax6 strongly changes the distribution of CR neurons along the depth of the developing cortex.

However, very little displacement from the pial surface was observed on sections (Fig. 4), suggesting that in culture we unmasked an effect which in the intact cortices is inhibited by possible cofactors not present in the cultures. To confirm that the changes observed in the Z-axis at E11.5 were not due to experimental conditions, cryostat sections of flattened cortices were prepared at the onset $(0 \mathrm{~h})$ and end $(8 \mathrm{~h})$ of the recording period (Supplementary Fig. S4A,B). The pial zone was identified by Reln-expressing cells and the VZ by Pax6-expressing cells in $\mathrm{M}$ and DL regions of control embryos (Supplementary Fig. S4C). After $8 \mathrm{~h}$ in culture, small changes were only detected in the $\mathrm{M}$ region where Reln ${ }^{+}$cells plunged deeper. In contrast, in DL regions the percentage of tissue occupied by Reln and Pax6 staining did not change between 0 and $8 \mathrm{~h}$, confirming that culture conditions do not alter distribution of CR cells along the $\mathrm{Z}$-axis of the developing neocortex. We confirmed that culture conditions did not affect the two genotypes differently after $8 \mathrm{~h}$ (data not shown; see Materials and Methods) and that cortex thickness was identical in controls and mutants. Finally, since $C R$ cell position was shown to influence migration of GABAergic neurons at the surface (MZ) and in the subventricular and intermediate zone (SVZ/IZ; Ang et al. 2003; Caronia-Brown and Grove 2011), we analyzed Calbindin (Calb) ${ }^{+}$interneurons in the DL neocortex of controls and mutants E13 embryos (Supplementary Fig. S5). We detected a decrease in the MZ and an increase in the SVZ/IZ at rostral but not caudal levels suggesting that defects in CR distribution correlate with defects in interneuron migration.

\section{Noncell Autonomous Effects on CR Cell Contact Repulsion}

CR cells, in particular CH-CR cells, were previously shown to migrate through a contact-repulsion mechanism controlling 
A

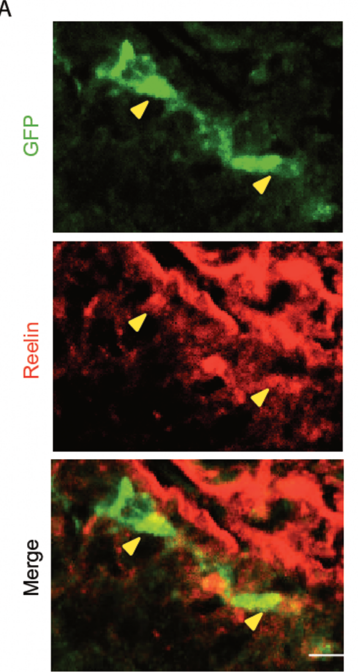

B

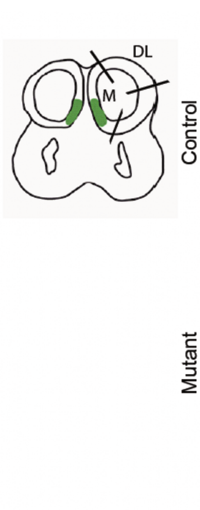

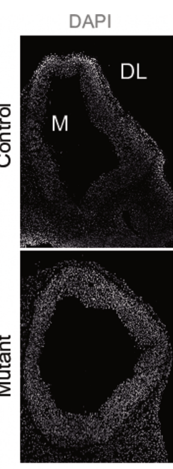
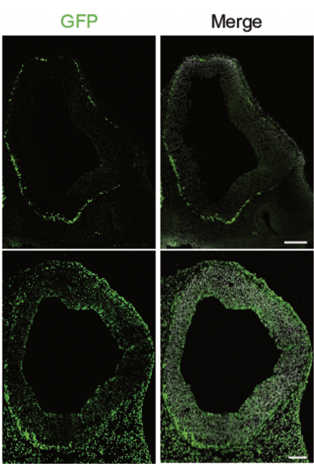

D

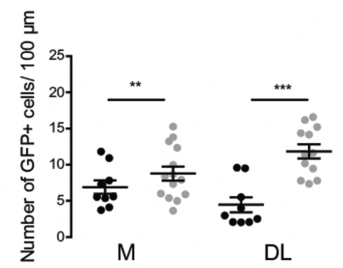

C

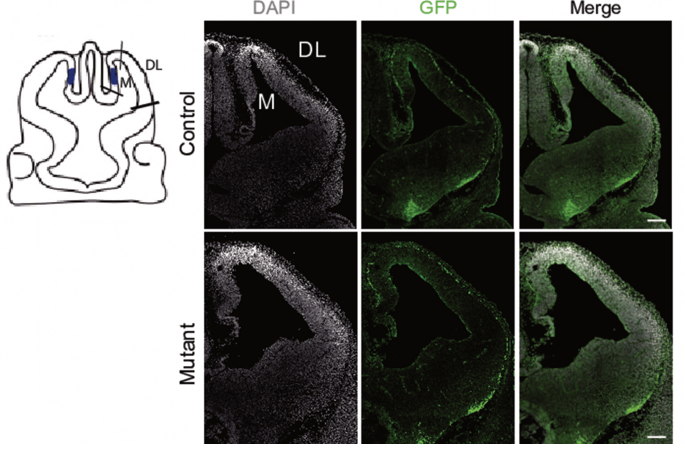

$\mathrm{E}$

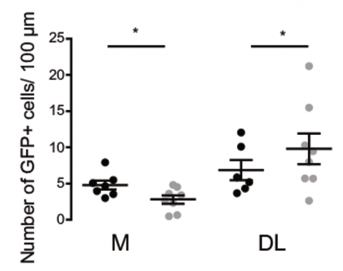

Figure 4. Pax6 neutralization alters CR neurons distribution in the lateral and rostral cortex. (A) High magnification of CR cells in the RL cortex from E11.5 mutants immunostained for GFP and Reln. CR neurons used for quantification were all Reln ${ }^{+} \mathrm{GFP}^{+}$(arrow). Scale bar: $10 \mu \mathrm{m}$. (B and C) Confocal images of coronal sections through the rostral (B) and caudal (C) telencephalon from E11.5 control and mutant embryos immunostained for DAPI and GFP. Scale bar: $100 \mu$ m. (D and E) Quantification at rostral (C) and caudal (D) levels along the rostral-caudal axis of the telencephalon of GFP ${ }^{+}$cells in $\mathrm{M}$ and DL regions. Black and gray dots show counts in control and mutant embryos, respectively. Graphical representation of normalized cell counts (cells/100 $\mu \mathrm{m} \pm \mathrm{SEM} ; n=3$ to seven brains for each genotype). Values shown are mean \pm SEM; ANOVA: $n s$ means not statistically different; ${ }^{*} \mathrm{P}<0.05 ;{ }^{* *} \mathrm{P}<0.01 ;{ }^{* * *} \mathrm{P}<0.001$.
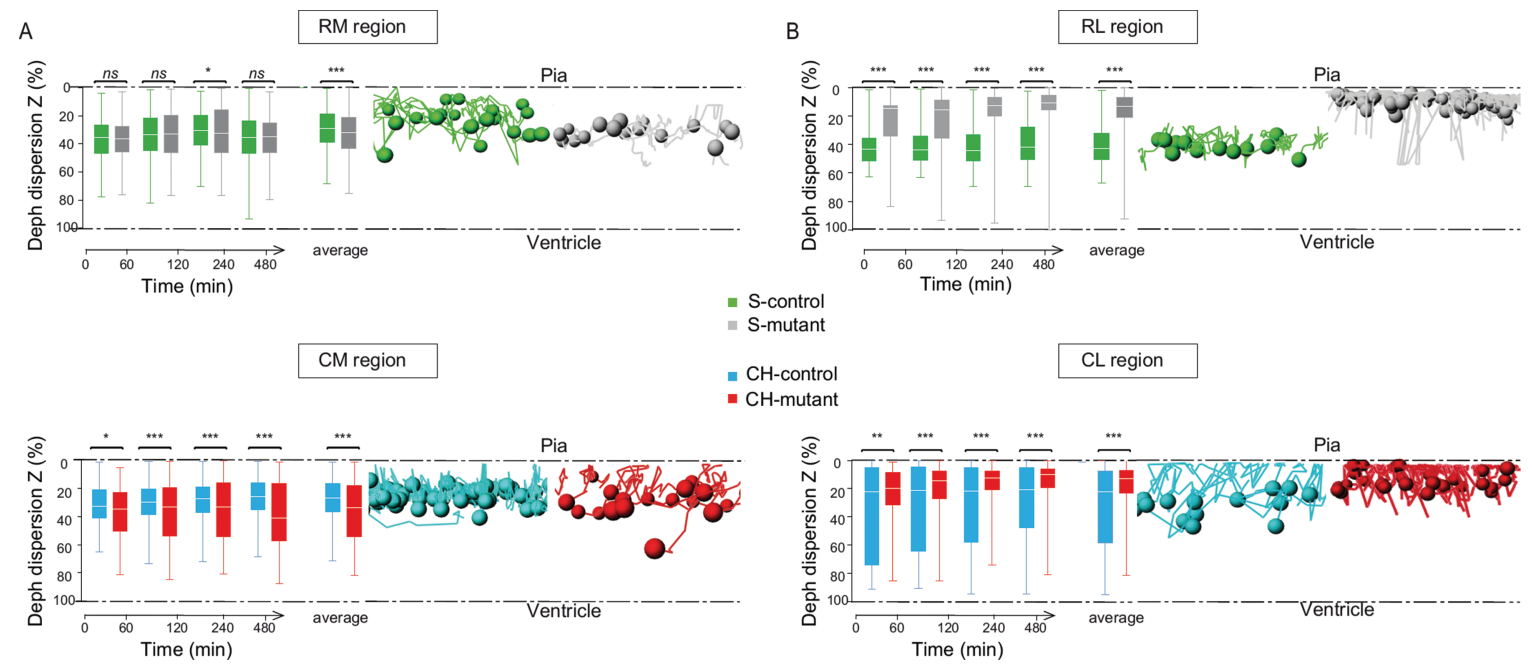

Figure 5. Blocking extracellular Pax6 affects CR cell depth. (A and B) Position of S- (A) and CH-CR (B) neurons in the depth axis (Z) for control and mutant cortices, classified by compartment at the onset of tracking (RM, RL, CM, and CL). For each cortex, values were weighted for comparison and are represented by percentage. $\mathrm{CR}$ cell localization is illustrated by representative $\mathrm{S}-$ and $\mathrm{CH}-\mathrm{CR}$ cells in each condition. Values shown are mean $\pm \mathrm{SEM}$; ANOVA: ns means not statistically different; ${ }^{*} \mathrm{P}<0.05 ;{ }^{* *} \mathrm{P}<0.01 ;{ }^{* * *} \mathrm{P}<0.001$.

their dispersion: when the growth cone of a CR cell comes into contact with another CR cell, its leading process retracts, its direction of migration changes by $>90^{\circ}$, and its migration speed increases (Villar-Cervino et al. 2013). In support of contact repulsion, we found that $\mathrm{CR}$ cells migrate faster at the onset of migration when cell density is higher and, in particular, $\mathrm{CH}-\mathrm{CR}$ cells displayed enhanced migration speed upon neutralization of extracellular Pax6 (Fig. 6A). After $1 \mathrm{hr}$ of recording, the speed was reduced for both CR cell subtypes with lower values recorded in mutants (Fig. 6A), suggesting that blocking extracellular Pax6 may have contrasting effects at the onset compared to later stages of migration. 
A

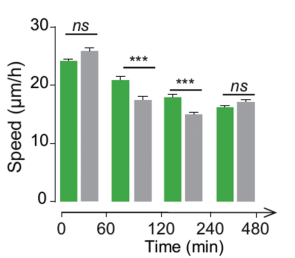

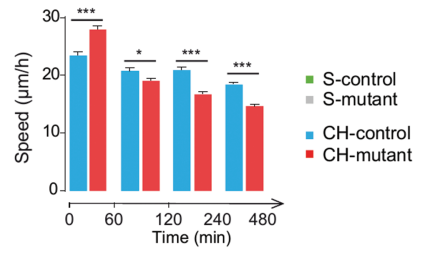

Hit contact

C

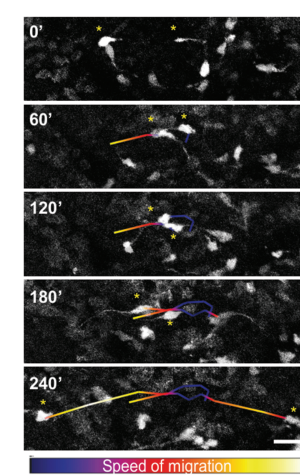

B
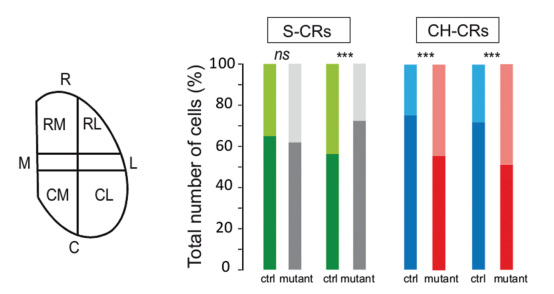

E
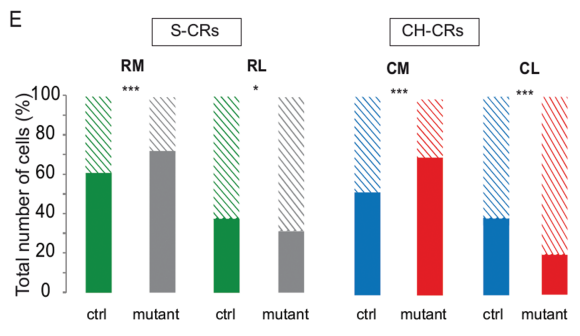

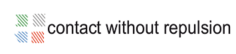
- contact-repulsion
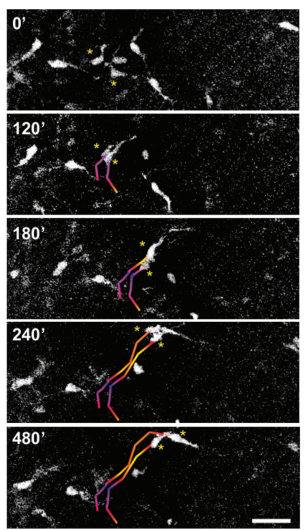

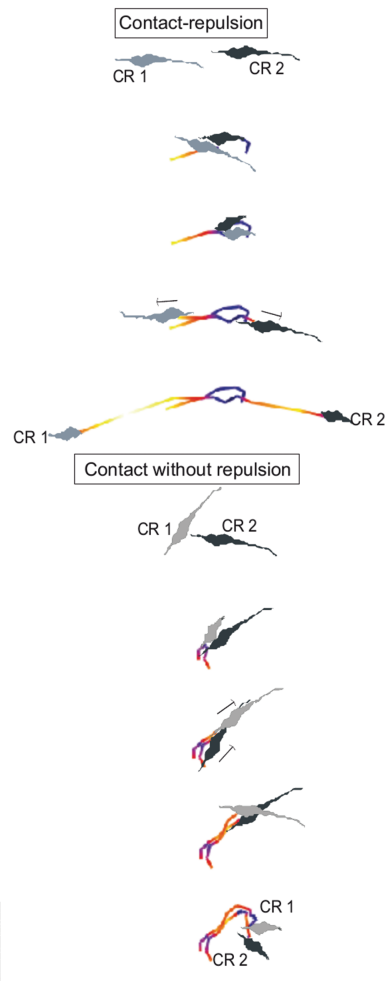

Figure 6. Pax6 neutralization stimulates or inhibits CR cell contact repulsion in a regions-dependent manner. (A) Speed of S- and CH-CR cells in controls and mutants were evaluated at different time points. All cells from the eight cortices were analyzed. (B) S- and CH-CR cells were clustered by "contact" if a CR cell encounters another CR cell during migration; "no contact" if a CR cell migrates without contacting another GFP ${ }^{+}$positive cell. (C-E) Contact between two cells was subdivided in two categories: $(C)$ "contact repulsion" when contact with another CR cell resulted in the full retraction of their leading process, re-extension of a new leading process, and change of initial direction (images taken from control CH-CR cells); and (D) "contact without repulsion" when contact with another CR cell did not change the direction (images taken from mutant S-CR cells). Tracks are color coded for speed of migration. Directionality of migration is represented by a white arrow. (E) Quantification of contact repulsion (filled bars) and contact without repulsion (hatched bars) of S- and CH-CR cells in control and mutant cortices. Values shown are mean \pm SEM; ANOVA: ns means not statistically different; ${ }^{*} \mathrm{P}<0.05 ;{ }^{* * *} \mathrm{P}<0.001$. Scale bar: $50 \mu \mathrm{m}$.

We therefore tested whether changes in contact repulsion may be involved in the migration defects observed in mutants. $\mathrm{GFP}^{+} \mathrm{CR}$ cells were clustered into two groups depending on whether or not they encounter and contact another $\mathrm{GFP}^{+} \mathrm{CR}$ cell during migration (Fig. 6B). In control conditions, S- and $\mathrm{CH}-\mathrm{CR}$ cells behaved differently, as $\mathrm{CH}-\mathrm{CR}$ cells displayed higher number of contacts in $\mathrm{M}$ and lateral domains compared to SCR cells $(74 \%$ in CL and $71 \%$ in CM vs $64 \%$ in RM and $56 \%$ in RL). Blocking extracellular Pax6 increased contact numbers between S-CR cells in the RL domain (73\% vs $64 \%$ ) and, on the contrary, decreased contact numbers in $\mathrm{CH}-\mathrm{CR}$ cells in both $\mathrm{CM}$ and CL territories (Fig. 6B). We further separated cells initiating contacts in two groups (Fig. 6C-E): 1) contact repulsion (Fig. 6C; movie S7) and 2) contact without repulsion when, following an encounter, cells remain in contact longer and do not change their direction of migration (Fig. 6D; movie S8). In control conditions, a higher percentage of both CR subtypes showed contact repulsion in $\mathrm{M}$ than in lateral domains confirming previous results that contact repulsion could be an important mechanism for cell dispersion near CR sources (Villar-Cervino et al. 2013). Blocking extracellular Pax6 enhanced contact repulsion in $\mathrm{M}$ regions and significantly diminished it in lateral regions (Fig. 6E). These differences were more marked for $\mathrm{CH}-\mathrm{CR}$ cells suggesting that neutralization of Pax 6 is more efficient in caudal-lateral regions. Together, these results show that extracellular Pax6 influence CR cells migration in a subtypespecific manner.

\section{Discussion}

In this study we used a genetic strategy to trigger the expression and secretion of a single-chain antibody against Pax6 to neutralize it in the extracellular space in the developing cortex in order to study its noncell autonomous function during neuronal migration. We show that neutralizing this secreted transcription factor modifies the migration behavior of S- and $\mathrm{CH}-\mathrm{CR}$ cells along both the tangential and $\mathrm{Z}$ trajectories. This genetic strategy, validated earlier for Pax6, Engrailed, and Otx2 (Lesaffre et al. 2007; Wizenmann et al. 2009; Di Lullo et al. 2011; Layalle et al. 2011; Bernard et al. 2016), decreases both extracellular Pax6 and Pax6 internalization. Based on these previous results, we favor the idea that noncell autonomous Pax6 activity requires its internalization to cause changes at the transcriptional, translational, and epigenetic levels. However, we cannot preclude a pure extracellular effect triggered by Pax6 extracellular binding sites or receptors.

Characterizing the factors which control correct positioning of $C R$ neurons at the cortical surface is a crucial issue since perturbing CR subtype distribution and migration alter the position and size of primary (Griveau et al. 2010) and higher-order areas 
(Barber et al. 2015). Using live imaging and genetic labeling, we followed S- and $\mathrm{CH}-\mathrm{CR}$ cells during early phases of migration. Upon extracellular Pax6 neutralization, we observed similarities and differences in the responses of the two CR subtypes. In terms of path length and Euclidian distances, neutralizing Pax6 had a negative effect, although stronger on Euclidian distance, both on S-CR and $\mathrm{CH}-\mathrm{CR}$ cells migrating with an RL direction toward the high concentration of Pax6 expression. Notably, this had no effect on S-CR cells whose tracks were oriented along the CL direction away from the high Pax 6 concentration. In terms of directionality, S-CR and $\mathrm{CH}-\mathrm{CR}$ cells responded similarly with a strong tendency to migrate more rostro-laterally in the mutant, an effect particularly striking for S-CR cells in the lateral region, suggesting that repulsive activity is stronger where Pax6 expression is higher (Manuel et al. 2015). These migration defects altered the distribution of $\mathrm{S}-$ and $\mathrm{CH}-\mathrm{CRs}$, which were more abundant in rostral and lateral regions of mutants compared to controls. Consistently, we also detected defects in interneuron migration in rostral regions. Although we cannot exclude that extracellular Pax6 has a direct effect on interneuron migration, these results support the cell non-autonomous role for this transcription factor in neuronal migration.

Given that for both subtypes the effect of Pax6 neutralization on Euclidian distance is stronger than on path length and that a reduction in directionality index is observed without changes in average migration speed, the phenotype reflects a disorganization of migration with little effect on the ability to migrate. Wild-type $\mathrm{CH}-\mathrm{CR}$ cells were previously found to migrate by expanded routes in Sey mutants embryos, in which both intracellular and extracellular Pax6 are eliminated, compared to control embryos (Ceci et al. 2010). They appeared to travel longer distances and to disperse more broadly along the rostrocaudal and Z-axis. Interestingly, we observed that CR cells of both subtypes traveled shorter distances and dispersed less, on both axes, upon specific removal of extracellular Pax6. This difference is best explained by the fact that, in contrast with the Sey mutant, neutralizing Pax6 noncell autonomous signaling functions leaves intact its cell autonomous transcriptional activities, suggesting a specific function of secreted Pax6 on CR cell migration. We also find that the effect on CR cell directionality upon neutralization of secreted Pax 6 depends on cell position at the onset of recording and that migration direction along the rostro-caudal axis is more highly disturbed in lateral regions, where Pax6 is expressed at higher levels. A possible explanation is that in lateral compartments, where Pax6 concentration is higher rostrally, neutralization of Pax6 extracellular activity redirects the cells toward a rostral direction, suggesting a repelling activity of high extracellular Pax6 concentrations. This dose- and region-dependent activity suggests that the graded intracellular Pax6 distribution in the embryonic cortex is preserved in the extracellular space and that secreted Pax6 is poorly diffusible. Indeed, extracellular HPs, as also found for several classical morphogens (Prochiantz and Di Nardo 2015), are unable to diffuse over long distances when not within complexes, and thus remain bound to the cell surface, probably trapped in the local extracellular matrix. This was confirmed for Engrailed in the optic tectum (Wizenmann et al. 2009) or in the Drosophila wing disk (Layalle S et al. 2011) and for Pax6 in the ventral neural tube (Di Lullo et al. 2011).

The analysis of CR cell dispersion within the neuroepithelium (Z-axis) reveals some differences in the effect of Pax6 neutralization between S-CR and CH-CR cells and also between territories. In the $\mathrm{M}$ compartments (RM and $\mathrm{CM}$ ), neutralizing
Pax6 had little influence on migration in the Z-axis, although a wider dispersion was noticed for $\mathrm{CH}-\mathrm{CR}$ cells in the mutant. In the lateral compartments, both S-CR and $\mathrm{CH}-\mathrm{CR}$ cells migrated closer to the surface in the mutant than in the wild type. This effect was milder for $\mathrm{CH}-\mathrm{CR}$ cells since control cells navigated more randomly dispersed along the Z-axis. Secreted Pax 6 could control CR cell dispersion in the Z-axis either directly on CR cells themselves or indirectly, through the alteration of RG, the end feet of which populate the marginal zone where CR cells migrate. It has also been proposed that chemokines such as CXCL12 secreted by the meninges not only enhance $C R$ cell motility but also maintain CR cells close to the pial surface (Borrell and Marin 2006). Our results thus suggest that extracellular Pax6 and CXCL12 may have opposite effects on the migration of CR cells in the Z-axis and on their maintenance in the marginal zone.

Contact repulsion was shown to be an important mechanism by which $\mathrm{CR}$ cells populate the cortex at early stages. In live-imaging studies, CR cells are repelled upon contact with neighbor cells resulting in the collapse and retraction of their leading process and a change in their direction of migration (Villar-Cervino et al. 2013; Barber et al. 2015). It was also shown that a proportion of CR neurons randomly change their migration direction without contacting adjacent cells (Villar-Cervino et al. 2013). For S-CR cells, neutralizing Pax6 increased the number of contacts in the RL but not in the RM compartment, although contacts were more often followed by repulsion in the latter compartment. For $\mathrm{CH}-\mathrm{CR}$ cells, we found a decrease in the number of contacts in the $\mathrm{CM}$ and CL with increased and decreased repulsion in the CM and CL compartments, respectively. We do not find a systematic correlation between contact and repulsion, suggesting that blocking extracellular Pax6 affects spontaneous changes in CR migration rather than contact repulsion. However, Eph/ephrin signaling was previously shown to be involved in contact repulsion. Abrogating this pathway possibly increased the duration of $\mathrm{CH}-\mathrm{CR}$ cell contact, thus reducing their ability to undergo contact repulsion (Villar-Cervino et al. 2013). Furthermore, we underscore the similarity between CR cell guidance by Pax6 and retinal growth cone guidance by Engrailed, which raises the possibility that, similarly to what was demonstrated for Engrailed (Wizenmann et al. 2009), Pax6 co-signals with Ephrins and/or other bona fide morphogens, including chemokines. Indeed, the possible differences observed in CR maintenance along the $\mathrm{Z}$-axis in cultures versus intact brains might also suggest that, although whole flattened cortical preparation are the closest possible to the in vivo situation, they lack a needed co-factor.

Altogether, our findings show for the first time that extracellular Pax6 controls CR neuron migration noncell autonomously, an observation analogous to the paracrine activity of Pax6 in oligodendrocyte precursor cell migration in the embryonic chick spinal cord (Di Lullo et al. 2011). This supports the hypothesis that several mechanisms can be employed by a gene/protein to execute the same function. We suggest that Pax6 may control cortical arealization by directly acting as a transcription factor in neuronal progenitors and indirectly as a guidance factor for proper $\mathrm{CR}$ cell migration. Therefore, slight changes in the levels and distribution of extracellular Pax6 during brain development could have important consequences in physiopathology and be at the origin of evolutionary modifications in the mammalian cerebral cortex. 


\section{Supplementary Material}

Supplementary material is available at Cerebral Cortex online.

\section{Funding}

Agence National de la Recherche (ANR)-Ministry of Science and Technology (MOST) Bilateral Accord (ANR-13-ISV2-0001 to A. Prochiantz and A. Pierani); European Research Council (ERC) Advanced Grant HOMEOSIGN (339379 to A. Prochiantz); ANR (ANR-2011-BSV4-023-01 and ANR-15-CE16-0003-01 to A. Pierani); Fondation Recherche Médicale (FRM) («Equipe FRM DEQ20130326521» to A. Pierani).

\section{Notes}

We thank the ImagoSeine facility, member of the France BioImaging infrastructure supported by the French National Research Agency (ANR-10-INSB-04, "Investments for the future"), for help with confocal microscopy and Imaris software. We thank the Buffon Animal facility, Animalliance and L. Vigier for technical assistance and animal care, F. Tissir for providing the $\triangle \mathrm{Np} 73^{\text {CrelRESGFP }}$ mouse line (Brussels, Belgium), F. Lam and V. Contremoulins for help with movie reconstruction and $\mathrm{O}$. Beaudoin for help with the biotinylation assay. Conflict of Interest: None declared.

\section{Authors' Contributions}

H.K. designed and performed most of the experiments; A.W., C.L.P., and P.M. participated in several experiments; M.V. and A.A.D. produced and validated the scFuPax6 mouse line; E.C. helped analyzing the data; A.P. and A.P. supervised the project and analyzed data; H.K., E.C., A.A.D., A.P., and A.P. wrote the manuscript.

\section{References}

Ang ES, Haydar TF, Gluncic V, Rakic P. 2003. Four-dimensional migratory coordinates of GABAergic interneurons in the developing mouse cortex. J Neurosci. 23:5805-5815.

Barber M, Arai Y, Morishita Y, Vigier L, Causeret F, Borello U, Ledonne F, Coppola E, Contremoulins V, Pfrieger FW et al. 2015. Migration speed of Cajal-Retzius cells modulated by vesicular trafficking controls the size of higher-order cortical areas. Curr Biol. 25:2466-2478.

Bernard C, Vincent C, Testa D, Bertini E, Ribot J, Di Nar do AA, Volovitch M, Prochiantz A. 2016. A mouse model for conditional secretion of specific single-chain antibodies provides genetic evidence for regulation of cortical plasticity by a non-cell autonomous homeoprotein transcription factor. PLoS Genet. 12:e1006035.

Bielle F, Griveau A, Narboux-Neme N, Vigneau S, Sigrist M, Arber S, Wassef M, Pierani A. 2005. Multiple origins of Cajal-Retzius cells at the borders of the developing pallium. Nat Neurosci. 8:1002-1012.

Borrell V, Marin O. 2006. Meninges control tangential migration of hem-derived Cajal-Retzius cells via CXCL12/CXCR4 signaling. Nat Neurosci. 9:1284-1293.

Brunet I, Di Nardo AA, Sonnier L, Beurdeley M, Prochiantz A. 2007. The topological role of homeoproteins in the developing central nervous system. Trends Neurosci. 30:260-267.
Brunet I, Weinl C, Piper M, Trembleau A, Volovitch M, Harris W, Prochiantz A, Holt C. 2005. The transcription factor Engrailed2 guides retinal axons. Nature. 438:94-98.

Caronia-Brown G, Grove EA. 2011. Timing of cortical interneuron migration is influenced by the cortical hem. Cereb Cortex. 21:748-755.

Ceci ML, Lopez-Mascaraque L, de Carlos JA. 2010. The influence of the environment on Cajal-Retzius cell migration. Cereb Cortex. 20:2348-2360.

Di Lullo E, Haton C, Le Poupon C, Volovitch M, Joliot A, Thomas JL, Prochiantz A. 2011. Paracrine Pax6 activity regulates oligodendrocyte precursor cell migration in the chick embryonic neural tube. Development. 138:4991-5001.

Di Nardo AA, Fuchs J, Joshi RL, Moya KL, Prochiantz A. 2018. The physiology of homeoprotein transduction. Physiol Rev. 98:1943-1982.

Garcia-Moreno F, Lopez-Mascaraque L, De Carlos JA. 2007. Origins and migratory routes of murine Cajal-Retzius cells. J Comp Neurol. 500:419-432.

Griveau A, Borello U, Causeret F, Tissir F, Boggetto N, Karaz S, Pierani A. 2010. A novel role for Dbx1-derived Cajal-Retzius cells in early regionalization of the cerebral cortical neuroepithelium. PLoS Biol. 8:e1000440.

Holcman D, Kasatkin V, Prochiantz A. 2007. Modeling homeoprotein intercellular transfer unveils a parsimonious mechanism for gradient and boundary formation in early brain development. J Theor Biol. 249:503-517.

Joliot A, Prochiantz A. 2004. Transduction peptides: from technology to physiology. Nat Cell Biol. 6:189-196.

Layalle S, Volovitch M, Mugat B, Bonneaud N, Parmentier ML, Prochiantz A, Joliot A, Maschat F. 2011. Engrailed homeoprotein acts as a signaling molecule in the developing fly. Development. 138:2315-2323.

Lesaffre B, Joliot A, Prochiantz A, Volovitch M. 2007. Direct noncell autonomous Pax6 activity regulates eye development in the zebrafish. Neural Dev. 2:2.

Manuel MN, Mi D, Mason JO, Price DJ. 2015. Regulation of cerebral cortical neurogenesis by the Pax 6 transcription factor. Front Cell Neurosci. 9:70.

Marin-Padilla M. 1998. Cajal-Retzius cells and the development of the neocortex. Trends Neurosci. 21:64-71.

Matsuo T, Osumi-Yamashita N, Noji S, Ohuchi H, Koyama E, Myokai F, Matsuo N, Taniguchi S, Doi H, Iseki S et al. 1993. A mutation in the Pax- 6 gene in rat small eye is associated with impaired migration of midbrain crest cells. Nat Genet. 3:299-304

O'Leary DD, Chou SJ, Sahara S. 2007. Area patterning of the mammalian cortex. Neuron. 56:252-269.

Pierani A, Moran-Rivard L, Sunshine MJ, Littman DR, Goulding M, Jessell TM. 2001. Control of interneuron fate in the developing spinal cord by the progenitor homeodomain protein Dbx1. Neuron. 29:367-384.

Prochiantz A. 2000. Messenger proteins: homeoproteins, TAT and others. Curr Opin Cell Biol. 12:400-406.

Prochiantz A, Di Nardo AA. 2015. Homeoprotein signaling in the developing and adult nervous system. Neuron. 85: 911-925.

Quininao C, Prochiantz A, Touboul J. 2015. Local homeoprotein diffusion can stabilize boundaries generated by graded positional cues. Development. 142:1860-1868.

Stoykova A, Hatano O, Gruss P, Gotz M. 2003. Increase in reelinpositive cells in the marginal zone of Pax6 mutant mouse cortex. Cereb Cortex. 13:560-571. 
Tissir F, Ravni A, Achouri Y, Riethmacher D, Meyer G, Goffinet AM. 2009. DeltaNp73 regulates neuronal survival in vivo. Proc Natl Acad Sci U S A. 106:16871-16876.

Villar-Cervino V, Molano-Mazon M, Catchpole T, Valdeolmillos M, Henkemeyer M, Martinez LM, Borrell V, Marin O. 2013. Contact repulsion controls the dispersion and final distribution of Cajal-Retzius cells. Neuron. 77:457-471.
Wizenmann A, Brunet I, Lam J, Sonnier L, Beurdeley M, Zarbalis K, Weisenhorn-Vogt D, Weinl C, Dwivedy A, Joliot A et al. 2009. Extracellular Engrailed participates in the topographic guidance of retinal axons in vivo. Neuron. 64:355-366.

Yoshida M, Assimacopoulos S, Jones KR, Grove EA. 2006. Massive loss of Cajal-Retzius cells does not disrupt neocortical layer order. Development. 133:537-545. 